\title{
A century of ferroelectricity
}

\author{
Ferroelectricity was experimentally discovered one hundred years ago, spurring research on its fundamental
} properties and potential applications.

U ltrasound, a commonly used medical procedure, is generated by piezoelectric materials. In a piezoelectric, applied stress generates electric charge and applied electric field generates stress, while the best piezoelectrics, in terms of large response to electric field, are switchable materials known as ferroelectrics. Below a critical temperature these possess a permanent electric polarization and, crucially in contrast to the wider class of piezeoelectrics, ferroelectric polarization can be reversed with the application of an electric field that is greater than the coercive field. The polarization reversal demonstrates hysteresis, or historydependent behaviour, while crystal structure changes during polarization switching. As indicated by the prefix 'ferro', ferroelectrics are analogous to earlier-known ferromagnets. This includes analogies such as permanent polarization to permanent magnetization, or the hysteretic nature of the relationship between the polarization (magnetization) and electric (or magnetic) field. Indeed, 2020 marks the 100th anniversary of the discovery, by a student, Joseph Valasek, of permanent polarization and hysteretic switching ${ }^{1}$.

Valasek performed an experimental study on Rochelle salt, potassium sodium tartrate tetrahydrate, which was known to exhibit piezoelectric behaviour. Valasek's research was driven by prior observations of the anomalous response of Rochelle salt to applied forces and electric fields, such as piezoelectric charge generation that deviates from the usual linear relationship with applied force, or dielectric properties varying with applied voltage. The confirmation of the permanent polarization and hysteresis effect on switching that are the defining characteristics of ferroelectrics was presented at a meeting of the American Physical Society in April 1920. This was followed up by the submission of a paper to the Physical Review in December that outlined how ferroelectrics are analogous to ferromagnets, and emphasized how Rochelle salt was different from other materials ${ }^{1}$.

This soon spurred interest in others to look for alternative ferroelectrics. Potassium dihydrogen phosphate, or KDP, was only discovered 13 years later by Paul Scherrer and Georg Busch and was found to exhibit a ferroelectric phase below $\sim 122 \mathrm{~K}$ (ref. ${ }^{2}$ ). However, like Rochelle salt, applications of KDP were initially limited, as both materials are fragile and water-soluble. It was only with

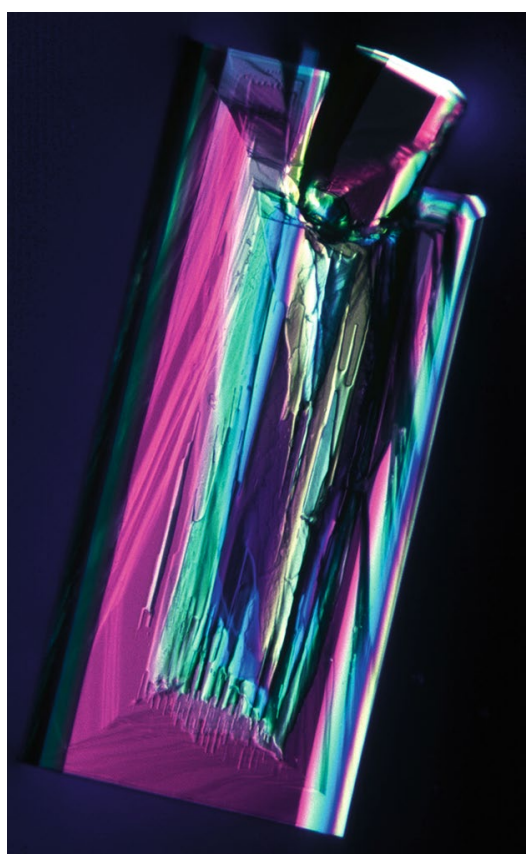

Rochelle salt under polarized light, showing evidence of different ferroelectric domains. Credit: Sciencephotos/Alamy Stock Photo

the discovery of robust ferroelectricity in a stable material, specifically $\mathrm{BaTiO}_{3}$ (ref. ${ }^{3}$ ), previously known to possess a high dielectric constant, that military and commercial application of ferroelectrics, in ultrasound devices as well as in other applications such as ferroelectric memories, could be considered ${ }^{4}$. The demonstration of ferroelectricity in an inorganic material spurred investigations into other perovskite oxides, eventually leading to the discovery of other materials such as $\mathrm{PbZrO}_{3}-\mathrm{PbTiO}_{3}$ compositions that are now used as piezoelectrics, or $\mathrm{BaTiO}_{3}$ derivatives that are used in capacitors. The simple structure of $\mathrm{BaTiO}_{3}$ allowed for detailed investigations of the crystal structure changes that take place through the ferroelectric phase transition, enabling the construction of the phenomenological model of ferroelectricity, as formulated by

A. F. Devonshire in the $1950 \mathrm{~s}^{5}$. This model describes the free energy of a ferroelectric material as a double-well potential with an energy barrier separating two polarization states. This has proven to be a powerful method for understanding ferroelectrics.
But by no means does this suggest that ferroelectrics research has stood still since then. Ferroelectrics remains a rich field of study. For example, in 1976 Rolf Landauer noted that as there is a free-energy barrier for ferroelectric switching there must be a region where the local curvature is negative, which means that in principle at that part of the freeenergy diagram capacitance is also negative ${ }^{6}$. It was much more recently understood that negative capacitance can be used to provide voltage amplification and was observed in thin ferroelectric films ${ }^{7}$. The double-well potential, the key to ferroelectric behaviour, was also recently observed using $\mathrm{Hf}_{0.5} \mathrm{Zr}_{0.5} \mathrm{O}_{2}$ (ref. ${ }^{8}$ ). This material shows no ferroelectricity in its bulk form, yet becomes a ferroelectric when it is in the form of a ferroelectric thin film 9 , indicating the role that improved growth methods can play. In this respect, in an Article in this issue by Darrell Schlom and colleagues, targeted chemical pressure at the atomic length scale is used to form $\left(\mathrm{SrTiO}_{3}\right)_{n-m}\left(\mathrm{BaTiO}_{3}\right)_{m} \mathrm{SrO}$ superlattices with a ferroelectric instability. This results in very low dielectric loss and tunability at frequencies of order $100 \mathrm{GHz}$, which may be relevant for microwave devices and wireless $5 \mathrm{G}$ applications - clearly far from any potential applications that might have been envisaged by Valasek.

The discovery of ferroelectricity is commonly assigned to 1920, but Valasek did not use that term throughout the paper. The modern concept of ferroelectricity was defined later, although at one point in his habilitation of 1912 Erwin Schrödinger used the phrase 'ferroelektrisch' in analogy to ferromagnetism for materials with permanent electric polarization. Since that initial analogy, and Valasek's pioneering observation of hysteresis, research and applications of these systems have proceeded at pace. We anticipate the second century of ferroelectricity will be as fruitful as the first.

\section{Published online: 27 January 2020}

https://doi.org/10.1038/s41563-020-0611-1

\section{References}

1. Valasek, J. Phys. Rev. 17, 475-481 (1921).

2. Scherrer, P. \& Busch, G. Naturwissenschaften 23, 737 (1935).

3. von Hippel, A., Breckenridge, A. V., Chesley, F. G. \& Tisza, L. Ind. Eng. Chem. 38, 1097-1109 (1946).

4. Scott, J. F. Science 315, 954-959 (2007).

5. Devonshire, A. F. Phil. Mag. 40, 1040-1063 (1949).

6. Landauer, R. Collect. Phenom. 2, 167-170 (1976).

Khan, A. I. et al. Nat. Mater. 14, 182-186 (2014).

8. Hoffmann, M. et al. Nature 565, 464-467 (2019).

9. Müller, J. et al. Appl. Phys. Lett. 99, 112901 (2011). 